\title{
Driving the discussion about the greater propensity for doing better with neoadjuvant chemotherapy for non-small cell lung cancer
}

\author{
Abigail K. Zamora, Anthony W. Kim \\ Division of Thoracic Surgery, Keck School of Medicine, University of Southern California, Los Angeles, CA, USA \\ Correspondence to: Anthony W. Kim, MD. Division of Thoracic Surgery, Keck School of Medicine, University of Southern California, 1510 San Pablo \\ St., Ste. 514, Los Angeles, CA 90033, USA. Email: Anthony.Kim@med.usc.edu. \\ Provenance: This is an invited article commissioned by the Academic Editor Dr. Shuangjiang Li (Department of Thoracic Surgery and West China \\ Medical Center, West China Hospital, Sichuan University, Chengdu, China). \\ Comment on: Brandt WS, Yan W, Zhou J, et al. Outcomes after neoadjuvant or adjuvant chemotherapy for cT2-4N0-1 non-small cell lung cancer: \\ A propensity-matched analysis. J Thorac Cardiovasc Surg 2019;157:743-53.e3.
}

Submitted Aug 21, 2019. Accepted for publication Sep 12, 2019.

doi: $10.21037 /$ jtd.2019.09.44

View this article at: http://dx.doi.org/10.21037/jtd.2019.09.44

One of the most challenging aspects of care in non-small cell lung cancer (NSCLC) is determining the optimal multimodal strategy to fight advanced disease at the time of presentation. The barrage of chemotherapy, radiation, surgery, and now immunotherapy may be beginning to mitigate the impact of locoregionally advanced disease on patient outcomes in NSCLC. In their important study "Outcomes after neoadjuvant or adjuvant chemotherapy for cT2-4N0-1 non-small cell lung cancer: a propensitymatched analysis" in The fournal of Thoracic and Cardiovascular Surgery Brandt et al. aimed to determine the impact of neoadjuvant chemotherapy (NC) versus adjuvant chemotherapy (AC) on patient outcomes for cT2-4N0-1 NSCLC (1).

The current National Comprehensive Cancer Network (NCCN) Guidelines for cT2-4N0-1 NSCLC include complete surgical resection followed by AC; however, NC followed by surgical resection can be considered as an alternative strategy (2). As the authors point out, these recommendations reflect data showing that surgery followed by AC have improved overall survival (OS) compared to surgery alone in phase III randomized clinical trials and a pooled analysis (3-5). There are limited phase III head-to-head comparisons of NC followed by surgery versus surgery followed by AC versus surgery alone in advanced stage disease, most notably the NATCH trial (6). In this clinical trial, the investigators found no difference in disease-free survival (DFS). The study cohort was comprised of a large proportion of patients with early stage disease. When performing a subgroup analysis of patients with higher stage lesions, those patients with clinical stage II (T3N1) disease were, in fact, observed to benefit from chemotherapy, regardless of its preoperative or postoperative administration (6).

In their study, Brandt et al. explored the issue of whether either $\mathrm{NC}$ or AC is better for patients with cT24N01 disease through a propensity-matched analysis. Their primary outcome was DFS, and their secondary outcomes were OS and chemotherapy regimen compliance. They performed a retrospective analysis of 330 patients with cT24N01 NSCLC over a 15-year period at their single tertiary care institution. Propensity-matching was performed resulting in 92 matched pairs with an absolute standardized mean difference (ASMD) $<0.1$ for each matched variable, confirming a good quality match. There was no difference between the NC and AC groups with respect to their primary outcome of DFS and one of their secondary outcomes, OS. However, NC was better tolerated, with patients completing more full cycles of chemotherapy with fewer severe side effects when compared to those receiving AC. While no direct improvement in patient outcomes was demonstrated, the authors' study highlighted several unique aspects and important issues.

Among the many strong elements to their study, the fact 
A Actual

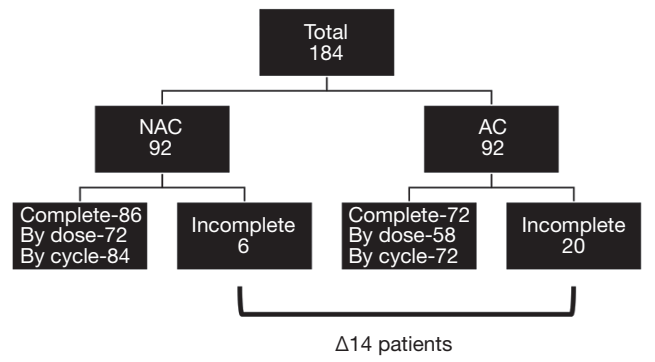

B Hypothetical

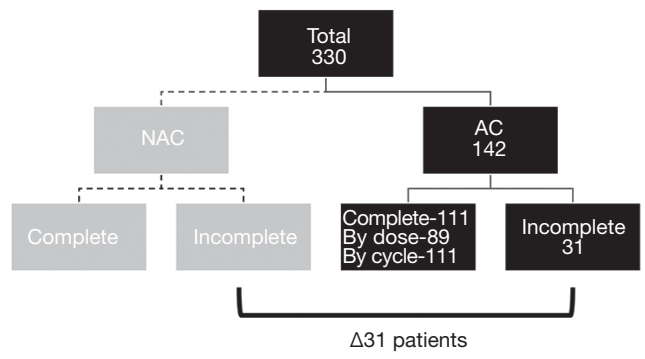

Figure 1 A hypothetical model demonstrating the impact of variable compliance associated with timing of systemic chemotherapy administration. Based upon the propensity-matched data, 13-15\% of patients with cT24N01 will not receive the benefit of chemotherapy if it is administered postoperatively. (A) Demonstrates the differences in chemotherapy compliance between the NC and AC cohorts according to the propensity-matched numbers reported; (B) demonstrates the increased number of patients unable to undergo chemotherapy when all patients previously undergoing $\mathrm{NC}$ are relegated to the AC cohort in a hypothetical model that uses the same proportions as the AC group in the propensity-matched cohort applied to the entire unmatched cohort. In this hypothetical model using the entire cohort of 330 patients, an additional 31 patients would not undergo systemic therapy if $\mathrm{AC}$ was the only option; whereas, this difference was 16 patients between the propensity-matched cohorts. NC, neoadjuvant chemotherapy; AC, adjuvant chemotherapy.

that their study cohort was comprised of a unique group of patients who were deemed to have locoregionally advanced disease without mediastinal lymph node involvement is very important. This group is often clustered with other locoregionally advanced cohorts that for many reasons may represent a different biologic form of disease. In point of fact, by virtue of their categorization into the larger group of patients with locoregionally advanced disease, the standards for therapy that may apply to the latter may not specifically apply to them, the former. Others have attempted to demonstrate this as well $(7,8)$. Simply put, their findings pertaining to the sequence of chemotherapy relative to surgery corroborate the notion that staging alone should not drive therapy.

By using a propensity-matched analysis, they limited bias and minimized confounding elements. Although, prospective, randomized clinical trials are a goal standard, developing a clinical trial to address the issues the authors have raised would be limited by challenges in accruing an adequate number of patients to achieve sufficient power to detect clinical differences. More importantly though as their own data suggests, there would be a true danger in not offering some form of chemotherapy to patients who would truly benefit from it. Perhaps another way to impart the strength of the study by Brandt and colleagues is to consider a hypothetical scenario in which the unmatched cohort is relegated to undergo AC. In the propensitymatched analysis, the AC arm received $13-15 \%$ fewer doses and cycles of chemotherapy than the NC arm (Figure 1). This percentage translated into a maximum of 6 and 21 patients who would fail to receive adequate systemic therapy in the NC and AC arms, respectively. In a hypothetical scenario of their entire cohort of 330 patients, if the 142 unmatched NC patients were now to undergo $\mathrm{AC}$, and the same distribution of patients who would not undergo AC is applied, 31 additional patients would fail to receive adequate systemic therapy. Given the substantial evidence demonstrating that chemotherapy associated with surgery is better than surgery alone in patients with advanced disease $(3,5,9-15)$, potentially depriving any patient in this cohort of chemotherapy would not meet a necessary standard.

Innumerable clinical trials in lung cancer as well as other malignancies have employed the Response Evaluation Criteria in Solid Tumors (RECIST) criteria based on computed tomography (CT) and positron emission tomography (PET) imaging to allow or disallow progression to surgical therapy following NC. The determination of its utility beyond this role, such as in predicting survival outcomes in the context of $\mathrm{NC}$, is an area that has been underappreciated. This oversight may be rooted in the belief that surgical therapy drives long term survival. However, the modern era of systemic therapy that is upon us suggests that this response may carry a greater gravitas that is similar in the degree of importance to a surgical intervention following $\mathrm{NC}$ and may prove to serve as a 
useful surrogate for longer-term survival-related outcomes. If nothing else, their observation that patients with an imaging response in the $\mathrm{NC}$ cohort had significantly improved DFS is very novel in the setting of cT24N01 disease.

While the RECIST criteria may provide some prognostic or predictive information, the current ability to predict major pathologic response (MPR) is limited. Their own data demonstrated that of the 14 patients who were deemed to have an MPR, imaging suggested stable disease in 5 patients and a partial response in 9 patients. Although it can be argued that downstaging may be a crude form of assessment of pathologic response, factoring into account MPR undoubtedly will provide a more granular level of data that could inform clinicians better regarding survival related outcomes $(16,17)$. Using MPR alone as an endpoint most assuredly will garner greater attention particularly as its ability to serve as a surrogate for longer-term survival outcomes gains momentum in modern era of therapy for NSCLC.

Although the authors performed sound analyses in their study, a few issues remain. While clearly a strength of their study is that they included a greater proportion of patients with unique form of advanced stage disease, discerning why some underwent $\mathrm{NC}$ versus $\mathrm{AC}$ remains a nuanced decision that will remain incompletely understood owing to the retrospective nature of this study. This decisionmaking process, in general, requires additional study for the purposes of enhancing clinical practice as it works two ways: deciding when those without nodal involvement should get $\mathrm{NC}$ or deciding when those with nodal involvement should undergo surgery first. Other points that remain unknown are whether or not the type of $\mathrm{NC}$ or AC chemotherapeutic agent or the timing of the administration of chemotherapy within the $\mathrm{NC}$ or AC settings have any impact on outcome measures. Another interesting issue that this study raises is that if there is no link between DFS and OS from a $\mathrm{NC}$ or AC standpoint, then one must consider whether or not guidelines should advocate for primary treatment with $\mathrm{NC}$ rather than $\mathrm{AC}$ given the potential advantages. The authors' findings demonstrating a significantly improved chemotherapy tolerance and adherence in the NC group make a strong case for this type of update. The superior compliance in the $\mathrm{NC}$ cohort is consistent with other prominent phase III trials and meta-analyses $(6,14)$. Secondary benefits would include the allowance for the assessment of tumor response prior to surgical resection as well as the ascertainment of more information to guide clinical decision-making as aforementioned.

In the end, Brandt and colleagues have performed an outstanding retrospective propensity-matched analysis of NC versus AC for cT2-4N0-1 NSCLC that provides the substrate for thoughtful discourse on the secondary benefits of NC. Thought provoking studies such as theirs are incredibly important as it forces all of us to reconsider convention. Furthermore, as the authors astutely point out, the NC versus AC debate most certainly will warrant greater interest as the data on immune checkpoint inhibitors and immunotherapy grows in NSCLC (18-22). For the time being, studies such as the one by Brandt et al. serve as a wonderful foundation to foster this discussion.

\section{Acknowledgments}

AW Kim has received travel and lodging funding from Roche-Genentech as a study steering committee member.

\section{Footnote}

Conflicts of Interest: The authors have no conflicts of interest to declare.

Ethical Statement: The authors are accountable for all aspects of the work in ensuring that questions related to the accuracy or integrity of any part of the work are appropriately investigated and resolved.

\section{References}

1. Brandt WS, Yan W, Zhou J, et al. Outcomes after neoadjuvant or adjuvant chemotherapy for cT2-4N0-1 non-small cell lung cancer: a propensity-matched analysis. J Thorac Cardiovasc Surg 2019;157:743-53.e3.

2. National Comprehensive Cancer Network. Non-small cell lung cancer (Version 5.2019). Accessed July 1, 2019. Available online: https://www.nccn.org/professionals/ physician_gls/PDF/nscl.pdf

3. Pignon JP, Tribodet H, Scagliotti GV, et al. Lung adjuvant cisplatin evaluation: a pooled analysis by the LACE Collaborative Group. J Clin Oncol 2008;26:3552-9.

4. Berry MF, Coleman BK, Curtis LH, et al. Benefit of adjuvant chemotherapy after resection of stage II (T12N1M0) non-small cell lung cancer in elderly patients. Ann Surg Oncol 2015;22:642-8.

5. Arriagada R, Bergman B, Dunant A, et al. Cisplatinbased adjuvant chemotherapy in patients with completely 
resected non-small cell lung cancer. $\mathrm{N}$ Engl J Med 2004;350:351-60.

6. Felip E, Rosell R, Maestre JA, et al. Preoperative chemotherapy plus surgery versus surgery plus adjuvant chemotherapy versus surgery alone in early-stage nonsmall-cell lung cancer. J Clin Oncol 2010;28:3138-45.

7. Moreno AC, Morgensztern D, Boffa DJ, et al. Treating locally advanced disease: an analysis of very large, hilar lymph node positive non-small cell lung cancer using the National Cancer Data Base. Ann Thorac Surg 2014;97:1149-55.

8. Gao SJ, Corso CD, Blasberg JD, et al. Role of Adjuvant Therapy for Node-Negative Lung Cancer Invading the Chest Wall. Clin Lung Cancer 2017;18:169-77.e4.

9. Thomas M, Rube C, Hoffknecht P, et al. Effect of preoperative chemoradiation in addition to preoperative chemotherapy: a randomized trial in stage III non-smallcell lung cancer. Lancet Oncol 2008;9:636-48.

10. Boffa DJ, Hancock JG, Yao X, et al. Now or later: evaluating the importance of chemotherapy timing in resectable stage III (N2) lung cancer in the National Cancer Database. Ann Thorac Surg 2015;99:200-8.

11. Pisters KM, Vallieres E, Crowley JJ, et al. Surgery with or without preoperative paclitaxel and carboplatin in earlystage non-small-cell lung cancer: Southwest Oncology Group Trial S9900, an intergroup, randomized, phase III trial. J Clin Oncol 2010;28:1843-9.

12. Scagliotti GV, Pastorino U, Vansteenkiste JF, et al. Randomized phase III study of surgery alone or surgery plus preoperative cisplatin and gemcitabine in stages IB to IIIA non-small-cell lung cancer. J Clin Oncol 2012;30:172-8.

13. Lim E, Harris G, Patel A, et al. Preoperative versus postoperative chemotherapy in patients with resectable non-small cell lung cancer: a systematic review and indirect comparison meta-analysis of randomized trials. J Thorac Oncol 2009;4:1380-8.

14. NSCLC Meta-analysis Collaborative Group. Preoperative chemotherapy for non-small-cell lung cancer: a systematic review and meta-analysis of individual participant data. Lancet 2014;383:1561-71.

15. Rosell R, Gomez-Codina J, Camps C, et al. A randomized trial comparing preoperative chemotherapy plus surgery with surgery alone in patients with non-small-cell lung cancer. N Engl J Med 1994;330:153-8.

16. Pataer A, Kalhor N, Correa AM, et al. Histopathologic Response Criteria Predict Survival of Patients with Resected Lung Cancer After Neoadjuvant Chemotherapy. J Thorac Oncol 2012;7:825-32.

17. Hellmann MD, Chaft JE, William WN Jr, et al. Pathological response after neoadjuvant chemotherapy in resectable non-small-cell lung cancers: proposal for the use of major pathological response as a surrogate endpoint. Lancet Oncol 2014;15:e42-50.

18. Ackermann CJ, Reck M, Paz-Ares L, et al. First-line immune checkpoint blockade for advanced non-small-cell lung cancer: travelling at the speed of light. Lung Cancer 2019;134:245-53.

19. Dafni U, Tsourti Z, Vervita K, et al. Immune checkpoint inhibitors, alone or in combination with chemotherapy, as first-line treatment for advanced non-small cell lung cancer. A systematic review and network meta-analysis. Lung Cancer 2019;134:127-40.

20. Mok TSK, Wu YL, Kudaba I, et al. Pembrolizumab versus chemotherapy for previously untreated, PD-L1expressing, locally advanced or metastatic non-small-cell lung cancer (KEYNOTE-042): a randomized, open-label, controlled phase 3 trial. Lancet 2019;393:1819-30.

21. Reck M, Rabe KF. Precision diagnosis and treatment for advanced non-small-cell lung cancer. N Engl J Med 2017;377:849-61.

22. Reck M, Rodriguez-Abreu D, Robinson AG, et al. Pembrolizumab versus chemotherapy for PD-L1positive non-small-cell lung cancer. N Engl J Med 2016;375:1823-33.
Cite this article as: Zamora AK, Kim AW. Driving the discussion about the greater propensity for doing better with neoadjuvant chemotherapy for non-small cell lung cancer. J Thorac Dis 2019;11(10):E174-E177. doi: 10.21037/ jtd.2019.09.44 